\begin{tabular}{|c|l|}
\hline Title & HOMOTOPY CLA SSIFICA TION OF NA NOPHRA SES IN TURAEV' STHEORY OF WORDS \\
\hline Author(s) & Fukunaga, Tomonori \\
\hline Citation & Hokkaido University Preprint Series in Mathematics, 897, 1-13 \\
\hline Issue Date & 2008 \\
\hline DOI & 10.14943/84047 \\
\hline Doc URL & http://hdl.handle.net/2115/69706 \\
\hline Type & bulletin (article) \\
\hline File Information & pre897.pdf \\
\hline
\end{tabular}

Instructions for use 


\title{
HOMOTOPY CLASSIFICATION OF NANOPHRASES IN TURAEV'S THEORY OF WORDS
}

\author{
FUKUNAGA Tomonori \\ Department of Mathematics, Hokkaido University \\ Sapporo 060-0810, Japan \\ e-mail: s063033@math.sci.hokudai.ac.jp
}

\begin{abstract}
The purpose of this paper is to give the homotopy classification of nanophrases of length 2 with 4 letters. To do it we construct some new invariants of nanophrases $\gamma, T$. The invariant $\gamma$ defined in this paper is an extension of the invariant $\gamma$ for nanowords introduced in [5]. The invariant $T$ is a new invariant of nanophrases. As a corollary of these results, we give the classification of two-components pointed, ordered, oriented curves on surfaces with minimum crossing number $\leq 2$.

Keywords: Words, Phrases, Curves, Homotopy
\end{abstract}

Mathematics Subject Classification 2000: 57M99, 68R15

\section{Introduction.}

Words are finite sequences of letters in a given alphabet. In [2] C. F. Gauss introduced a method to investigate closed planar curves by words of a certain type now called Gauss words. We can apply this method to encode surface curves. (See [10].)

V.Turaev introduced word theory in [5], [6]. The key of new concepts introduced in those papers are those of étale words and nanowords. An étale word over an alphabet $\alpha$ endowed with an involution $\tau: \alpha \longrightarrow \alpha$ is a word in an alphabet $\mathcal{A}$ endowed with a projection $\mathcal{A} \ni A \mapsto|A| \in \alpha$. Every word in the alphabet $\alpha$ becomes an étale word over $\alpha$ by using the identity mapping $i d: \alpha \longrightarrow \alpha$ as the projection. An étale word over $\alpha$ is called nanoword if every letter appears twice or not at all. In the case where the alphabet $\alpha$ consists of two elements permuted by $\tau$, the notion of a nanoword over $\alpha$ is equivalent to the notion of an open virtual string introduced in [9].

Turaev introduced an equivalence relation of homotopy on the set of étale words over $\alpha$. The relation of homotopy is generated by three transformations or moves on nanowords. The first move consists in deleting two consecutive entries of the same letter. The second move has the form $x A B y B A z \mapsto x y z$ where $x, y, z$ are words and $A, B$ are letters such that $|A|=\tau(|B|)$. The third move has the form $x A B y A C z B C t \mapsto x B A y C A z C B t$ where $x, y, z, t$ are words and $A, B, C$ are letters such that $|A|=|B|=|C|$. These moves are suggested by the Reidemeister moves 
in knot theory. In fact the first (resp. second, third) homotopy move is similar to the first (resp. second, third) Reidemeister move.(See [6] for more details). Turaev applied topological methods to a semigroup consisting letters to study properties and characteristics of nanowords preserved under homotopy. For instance,these are applications of colorings of knot diagrams, the theory of knot quandles, etc. (See [5], [6], [7], [4] for more details.) As an application of those methods, Turaev gave the homotopy classification of nanowords of length $\leq 6$ in [5].

On the other hand, in [6] Turaev showed that a stable equivalence class of an oriented pointed curve on a surface is identified with a homotopy class of nanoword in a 2-letter alphabet. Moreover Turaev extended this result to multi-component curves. In fact a stable equivalence class of an oriented, ordered, pointed multicomponent curve on a surface is identified with a homotopy classes of a nanophrase in a 2-letter alphabet. Roughly speaking, a nanophrase is a sequence of étale words which concatenation of those words is a nanoword. (See [6], [8].) We can define homotopy moves similarly as in the case of nanowords.

Now the purpose of this paper is to give the homotopy classification of nanophrases of length 2 with 4 letters. (Theorem 4.6.) To do it we construct some new invariants of nanophrases. As a corollary of these results, we give the classification of two-components pointed, ordered, oriented curves on surfaces with minimum crossing number $\leq 2$. (See also $[1]$.)

Another application of the theory of words was introduced by N.Ito in [3]. By using the theory of words, Ito reconstructed the Arnold basic invariants and constructed some other invariants for plane closed curves, long curves, and fronts.

In section 2 we review the theory of words and phrases which are introduced by Turaev in [5], [6]. In section 3 we construct some new homotopy invariants of nanophrases $\gamma, T$. The invariant $\gamma$ defined in this paper is an extension of the invariant $\gamma$ for nanowords introduced in [5]. The invariant $T$ is a new invariant of nanophrases. In section 4 we generalize Turaev's result to the case of nanophrases. In fact we give the homotopy classification of nanophrases of length 2 with 4 letters using homotopy invariants constructed in section 3 .

\section{Nanowords and Nanophrases.}

In this section we review the theory of words and phrases (cf.[5], [6]).

\subsection{Nanowords and their homotopy.}

An alphabet is a set and letters are its elements. A word of length $n \geq 1$ on an alphabet $\mathcal{A}$ is a mapping $w: \hat{n} \rightarrow \mathcal{A}$ where $\hat{n}=\{1,2, \cdots, n\}$. A word usually encoded by the sequence of letters $w(1) w(2) \cdots w(n)$. A word $w: \hat{n} \rightarrow \mathcal{A}$ is a Gauss word if each element of $\mathcal{A}$ is the image of precisely two elements of $\hat{n}$.

For a set $\alpha$, an $\alpha$-alphabet is a set $\mathcal{A}$ endowed with a mapping $\mathcal{A} \rightarrow \alpha$ called projection. the image of $A \in \mathcal{A}$ under this mapping is denoted $|A|$. A étale word 
over $\alpha$ is a pair (an $\alpha$-alphabet, a word on $\mathcal{A}$ ). A nanoword over $\alpha$ is a pair (an $\alpha$-alphabet, a Gauss word on $\alpha$ ). An empty étale word in an empty $\alpha$-alphabet is a nanoword called the empty nanoword $\emptyset$ of length 0 .

A morphism of $\alpha$-alphabets $\mathcal{A}_{1}, \mathcal{A}_{2}$ is a set-theoric mapping $f: \mathcal{A}_{1} \longrightarrow \mathcal{A}_{2}$ such that $|A|=|f(A)|$ for all $A \in \mathcal{A}_{1}$. If $f$ is bijective, then this morphism is an isomorphism. Two étale words $\left(\mathcal{A}_{1}, w_{1}\right)$ and $\left(\mathcal{A}_{2}, w_{2}\right)$ over $\alpha$ are isomorphic if there is an isomorphism $f: \mathcal{A}_{1} \longrightarrow \mathcal{A}_{2}$ such that $w_{2}=f \circ w_{1}$.

To define homotopy of nanowords we fix a set $\alpha$ with an involution $\tau: \alpha \longrightarrow \alpha$ and a subset $S \subset \alpha \times \alpha \times \alpha$. We call the pair $(\alpha, S)$ homotopy data.

Definition 2.1. Let $(\alpha, S)$ be homotopy data. We define a homotopy moves (1) (3) as follows:

(1) $(\mathcal{A}, x A A y) \longrightarrow(\mathcal{A} \backslash\{A\}, x y)$

for all $A \in \mathcal{A}$ and $x, y$ are words in $\mathcal{A} \backslash\{A\}$.

(2) $(\mathcal{A}, x A B y B A z) \longrightarrow(\mathcal{A} \backslash\{A, B\}, x y z)$

if $A, B \in \mathcal{A}$ with $|B|=\tau(|A|) . x, y, z$ are words in $\mathcal{A} \backslash\{A, B\}$.

(3) $(\mathcal{A}, x A B y A C z B C t) \longrightarrow(\mathcal{A}, x B A y C A z C B t)$

if $A, B, C \in \mathcal{A}$ satisfy $(|A|,|B|,|C|) \in S . x, y, z, t$ are words in $\mathcal{A}$.

Definition 2.2. Let $(\alpha, S)$ be a homotopy data. Then nanowords $\left(\mathcal{A}_{1}, w_{1}\right)$ and $\left(\mathcal{A}_{2}, w_{2}\right)$ over $\alpha$ are $S$-homotopic (denote $\left.\left(\mathcal{A}_{1}, w_{1}\right) \simeq_{S}\left(\mathcal{A}_{2}, w_{2}\right)\right)$ if $\left(\mathcal{A}_{2}, w_{2}\right)$ can be obtained from $\left(\mathcal{A}_{1}, w_{1}\right)$ by a finite sequence of isomorphism, $S$-homotopy moves (1)

- (3) and the inverse moves.

The set of $S$-homotopy classes of nanowords over $\alpha$ is denoted as $\mathcal{N}(\alpha, S)$.

To define $S$-homotopy of étale words. We define desingularization of étale words $(\mathcal{A}, w)$ over $\alpha$ as follows: $\mathcal{A}^{d}:=\left\{A_{i, j}:=(A, i, j) \mid A \in \mathcal{A}, 1 \leq i<j \leq m_{w}(A)\right\}$ with projection $\left|A_{i, j}\right|:=|A| \in \alpha$ for all $A, i, j$ (where $m_{w}(A):=\operatorname{Card}\left(w^{-1}(A)\right)$ ). The word $w^{d}$ is obtained from $w$ by first deleting all $A \in \mathcal{A}$ with $m_{w}(A)=1$. Then for each $A \in \mathcal{A}$ with $m_{w}(A) \geq 2$ and each $i=1,2, \ldots m_{w}(A)$, we replace the $i$-th entry of $A$ in $w$ by

$$
A_{1, i} A_{2, i} \ldots A_{i-1, i} A_{i, i+1} A_{i, i+2} \ldots A_{i, m_{w}(A)} .
$$

The resulting $\left(\mathcal{A}^{d}, w^{d}\right)$ is a nanoword of length $\Sigma m_{w}(A)\left(m_{w}(A)-1\right)$ and called a desingularization of $(\mathcal{A}, w)$. Then we define $S$-homotopy of étale words as following:

Definition 2.3. Let $w_{1}$ and $w_{2}$ be étale words over $\alpha$. Then $w_{1}$ and $w_{2}$ are $S$ homotopic if $w_{1}^{d}$ and $w_{2}^{d}$ are $S$-homotopic.

Recall the following three lemmas from [5].

Lemma 2.4. Let $(\alpha, S)$ be a homotopy data and $\mathcal{A}$ be an $\alpha$-alphabet. $A, B, C$ are distinct letters in $\mathcal{A}$. $x, y, z, w$ are words in $\mathcal{A} \backslash\{A, B, C\}$ with xyzt is Gauss word. Then following (i)-(iii) are hold:

(i) $(\mathcal{A}, x A B y C A z B C t) \simeq_{S}(\mathcal{A}, x A B y A C z C B t)$ 


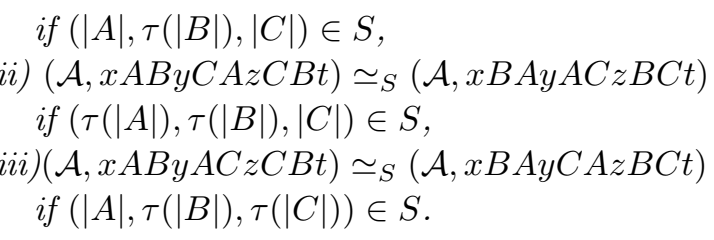

Lemma 2.5. Suppose that $S \cap(\alpha \times b \times b) \neq \emptyset$ for all $b \in \alpha$. Let $(\mathcal{A}, x A B y A B z)$ be nanoword over $\alpha$ with $|B|=\tau(|A|) . x, y, z$ are words in $\mathcal{A} \backslash\{A, B\}$.Then

$$
(\mathcal{A}, x A B y A B z) \simeq_{S}(\mathcal{A} \backslash\{A, B\}, x y z) .
$$

In the remaining part of the paper we assume that $S$ is the diagonal of $\alpha^{3}$ that is $\{(a, a, a)\}_{a \in \alpha}$. Under this convention, we shall omit the prefix $S$ - and speak simply of homotopy rather than $S$-homotopy. We shall also omit index $S$ and write $\simeq$, $\|\cdot\|$, $\mathcal{N}_{\bullet}(\alpha)$ for $\simeq_{S},\|\cdot\|_{S}, \mathcal{N}_{\bullet}^{S}(\alpha)$.

Lemma 2.6. Let $\beta$ be $\tau$-invariant subset of $\alpha$. If two étale words over $\beta$ are homotopic in the class of étale words over $\alpha$, then they are homotopic in the class of étale words over $\beta$.

V.Turaev gives a homotopy classification of nanowords of length 4 in [5].

Theorem 2.7. Let $w$ be a nanoword of length 4 over $\alpha$. Then $w$ is either $w \simeq \emptyset$ or isomorphic to the nanoword $w_{a, b}:=(\mathcal{A}=\{A, B\}, A B A B)$ where $|A|=a,|B|=$ $b \in \alpha$ with $a \neq \tau(b)$. Moreover for $a \neq \tau(b)$, the nanoword $w_{a, b}$ is non-contractible and two nanowords $w_{a, b}$ and $w_{a^{\prime}, b^{\prime}}$ are homotopic if and only if $(a, b)=\left(a^{\prime}, b^{\prime}\right)$.

In this paper we generalize Turaev's result to the case of "nanophrases".

\subsection{Nanophrases and their homotopy.}

Definition 2.8. A nanophrase $\left(\mathcal{A},\left(w_{1}\left|w_{2}\right| \cdots \mid w_{k}\right)\right)$ of length $k \geq 0$ over a set $\alpha$ is a pair consisting of an $\alpha$-alphabet $\mathcal{A}$ and a sequence of $k$ words $w_{1}, \cdots, w_{k}$ on $\mathcal{A}$ such that $w_{1} w_{2} \cdots w_{k}$ is a Gauss word on $\mathcal{A}$. We denote it shortly by $\left(w_{1}\left|w_{2}\right| \cdots \mid w_{k}\right)$. We denote a set of nanophrases of length $k$ over $\alpha$ by $\mathcal{P}_{k}(\alpha)$.

By definition, there is a unique empty nanophrase of length 0 (the corresponding $\alpha$-alphabet $\mathcal{A}$ is void).

Remark 2.9. Any nanoword $w$ over $\alpha$ yields a nanophrase $(w)$ of length 1 .

A mapping $f: \mathcal{A}_{1} \longrightarrow \mathcal{A}_{2}$ is isomorphism of two nanophrases if $f$ is an isomorphism of $\alpha$-alphabets transforming the first nanophrase into second one.

Given a homotopy data $(\alpha, \tau, S)$, we define homotopy move on nanophrases as in section 2.1 with the only difference that the 2-letter subwords $A A, A B, B A, A C$, $B C$ modified by these moves may occur in different words of phrase. Isomorphism 
and homotopy moves generate an equivalence relation $\simeq_{S}$ of $S$-homotopy on the class of nanophrases over $\alpha$. We denote a set of $S$-homotopy class of nanophrases of length $k$ by $\mathcal{P}_{k}(\alpha, S)$.

Example 2.10. Nanophrases $(A B \mid A D D C B C)$ and $(B A \mid C A C B)$ with $|A|=$ $|B|=|C| \in \alpha$ over $\alpha$ are homotopic. Indeed

$$
(A B \mid A \underline{D D} C B C) \simeq(\underline{A B} \mid \underline{A C B C}) \simeq(B A \mid C A C B) .
$$

Lemmas2.4 and 2.5 extend to nanophrases with the only change that the 2-letter subwords $A B, B A, C A$, and so forth may occur in different word of the phrase.

\section{Some Homotopy Invariants of Nanophrases.}

In this section, we define three new homotopy invariants of nanophrases. They will be used in the next section.

\subsection{Invariant $\gamma$.}

Recall that an orbit of the involution $\tau: \alpha \longrightarrow \alpha$ is a subset of $\alpha$ consisting either of one element or of two elements; in latter case the orbit is called free. Let $\Pi$ be the group which defined as follows:

$$
\Pi:=\left(\left\{z_{a}\right\}_{a \in \alpha} \mid z_{a} z_{\tau(a)}=1 \text { for all } a \in \alpha\right) .
$$

Let $\mathbb{Z} \Pi$ be the integral group-ring of $\Pi$.

Definition 3.1. Let $P=\left(\mathcal{A},\left(w_{1}\left|w_{2}\right| \cdots \mid w_{k}\right)\right)$ be a nanophrase of length $k$ over $\alpha$ and $n_{i}$ the length of nanoword $w_{i}$. Set $n=\sum_{1 \leq i \leq k} n_{i}$. Then we define $n$ elements $\gamma_{1}^{i}, \gamma_{2}^{i}, \cdots, \gamma_{n_{i}}^{i}(i \in\{1,2, \cdots, k\})$ of $\Pi$ by $\gamma_{i}^{j}:=z_{\left.\mid w_{j}(i)\right) \mid}$ if $w_{j}(i) \neq w_{l}(m)$ for all $l<j$ and for all $m<i$ when $l=j$. Otherwise $\gamma_{i}^{j}:=z_{\tau\left(\left|w_{j}(i)\right|\right)}$. Then we define $\gamma(P) \in \otimes^{k} \mathbb{Z} \Pi$ by

$$
\gamma(P):=\gamma_{1}^{1} \gamma_{2}^{1} \cdots \gamma_{n_{1}}^{1} \otimes \gamma_{1}^{2} \gamma_{2}^{2} \cdots \gamma_{n_{2}}^{2} \otimes \cdots \otimes \gamma_{1}^{k} \gamma_{2}^{k} \cdots \gamma_{n_{k}}^{k}
$$

Then we obtain following theorem.

Theorem 3.2. The $\gamma$ is a homotopy invariant of nanophrases.

Remark 3.3. By definition, for nanophrases of length 1 the invariant $\gamma$ for nanophrases is equal to Turaev's invariant $\gamma$ defined in [5].

Example 3.4. Let $\mathcal{A}:=\{A, B, C\}$ be an $\alpha$-alphabet. Set $|A|=a,|B|=b,|C|=$ $c \in \alpha$. Consider a nanophrase $P=(A B C|C B| A)$, then

$$
\gamma(P)=z_{a} z_{b} z_{c} \otimes z_{\tau(c)} z_{\tau(b)} \otimes z_{\tau(a)} .
$$




\subsection{Invariant $T$.}

In this subsection we define homotopy invariants of nanophrases over $\alpha_{0}:=\{a, b\}$ with involution $\tau_{0}$ permuting $a, b$ and nanophrases over one-point set. At first, we define a homotopy invariant of nanophrases $T$ over $\alpha_{0}$. To define this invariant, we define some notation as follows.

Definition 3.5. Let $P=\left(\mathcal{A},\left(w_{1}|\cdots| w_{k}\right)\right)$ be a nanophrase over $\alpha_{0}$ and $A, B \in \mathcal{A}$. Then we define $\sigma_{P}(A, B)$ as follows: If $A$ and $B$ form $\cdots A \cdots B \cdots A \cdots B \cdots$ in $P$ and $|B|=a$, or $\cdots B \cdots A \cdots B \cdots A \cdots$ in $P$ and $|B|=b$, then $\sigma_{P}(A, B):=1$. If $\cdots A \cdots B \cdots A \cdots B \cdots$ in $P$ and $|B|=b$, or $\cdots B \cdots A \cdots B \cdots A \cdots$ in $P$ and $|B|=a$, then $\sigma_{P}(A, B):=-1$. Otherwise $\sigma_{P}(A, B):=0$.

Definition 3.6. For $A \in \mathcal{A}$ we define $\varepsilon(A) \in\{ \pm 1\}$ by

$$
\varepsilon(A):=\left\{\begin{array}{l}
1(\text { if }|A|=a), \\
-1(\text { if }|A|=b) .
\end{array}\right.
$$

Definition 3.7. Let $P=\left(\mathcal{A},\left(w_{1}\left|w_{2}\right| \cdots \mid w_{k}\right)\right)$ be a nanophrase of length $k$ over $\alpha_{0}$. For $A \in \mathcal{A}$ such that there exist $i \in\{1,2, \cdots, k\}$ such that $\operatorname{Card}\left(w_{i}^{-1}(A)\right)=2$, we define $T_{P}(A) \in \mathbb{Z}$ by

$$
T_{P}(A):=\varepsilon(A) \sum_{B \in \mathcal{A}} \sigma_{P}(A, B),
$$

and we define $T_{P}\left(w_{i}\right) \in \mathbb{Z}$ by

$$
T_{P}\left(w_{i}\right):=\sum_{A \in \mathcal{A}, \operatorname{Card}\left(w_{i}^{-1}(A)\right)=2} T_{P}(A) .
$$

Then we define $T(P) \in \mathbb{Z}^{k}$ by

$$
T(P):=\left(T_{P}\left(w_{1}\right), T_{P}\left(w_{2}\right), \cdots, T_{P}\left(w_{k}\right)\right) .
$$

Theorem 3.8. The T is a homotopy invariant of nanophrases over $\alpha_{0}$.

Proof. Consider the 1-st homotopy move

$P_{1}:=\left(w_{1}|\cdots| w_{l-1}|x A A y| w_{l+1}|\cdots| w_{k}\right) \longrightarrow$

$$
P_{2}:=\left(w_{1}|\cdots| w_{l-1}|x y| w_{l+1}|\cdots| w_{k}\right) .
$$

It is clear that $T_{P_{1}}\left(w_{i}\right)=T_{P_{2}}\left(w_{i}\right)$ for all $i \neq l$. We show that $T_{P_{1}}(x A A y)=T_{P_{2}}(x y)$. Note that $\sigma_{P_{1}}(A, B)=0$ for all $B \in \mathcal{A}$ by definition. Therefore $T_{P_{1}}(A)=0$. Moreover $\sigma_{P_{1}}(E, A)=0$ for all $E \in \mathcal{A}$. So $A$ does not contribute to $T_{P_{1}}(E)$ for all $E \in \mathcal{A}$. Therefore $T_{P_{1}}(x A A y)=T_{P_{2}}(x y)$.

Consider the 2-nd homotopy move such that $\mathrm{A}$ and $\mathrm{B}$ occur in some words once $P_{1}:=\left(w_{1}|\cdots| x_{1} A B y_{1}|\cdots| x_{2} B A y_{2}|\cdots| w_{k}\right) \longrightarrow$

with $|A|=\tau(|B|)$.

$$
P_{2}:=\left(w_{1}|\cdots| x_{1} y_{1}|\cdots| x_{2} y_{2}|\cdots| w_{k}\right) .
$$


It is sufficient to show that $T_{P_{1}}\left(x_{1} A B y_{1}\right)=T_{P_{2}}\left(x_{1} y_{1}\right)$ and $T_{P_{1}}\left(x_{2} B A y_{2}\right)=$ $T_{P_{2}}\left(x_{2} y_{2}\right)$. Note that $A$ and $B$ occur in $P$ once. Moreover for all $E$ such that $\cdots E \cdots A B \cdots E \cdots$ in $P_{1}$

$$
\begin{aligned}
T_{P_{1}}(E) & =\varepsilon(E)\left(n_{1}+\sigma_{P_{1}}(E, A)+\sigma_{P_{1}}(E, B)+n_{2}\right) \\
& =\varepsilon(E)\left(n_{1}+n_{2}\right) \\
& =T_{P_{2}}(E)
\end{aligned}
$$

where $n_{1}, n_{2}$ are integers. Therefore $T_{P_{1}}\left(x_{1} A B y_{1}\right)=T_{P_{2}}\left(x_{1} y_{1}\right) . T_{P_{1}}\left(x_{2} B A y_{2}\right)=$ $T_{P_{2}}\left(x_{2} y_{2}\right)$ is proved similarly.

Consider the 2-nd homotopy move such that $A$ and $B$ occur in some word twice $P_{1}:=\left(w_{1}|\cdots| w_{l-1}|x A B y B A z| w_{l+1}|\cdots| w_{k}\right) \longrightarrow P_{2}:=\left(w_{1}|\cdots| x y z|\cdots| w_{k}\right)$ with $|A|=\tau(|B|)$. It is sufficient to show that $T_{P_{1}}\left(w_{l}\right)=T_{P_{2}}\left(w_{l}\right)$. At first we show $T_{P_{1}}(A)+T_{P_{1}}(B)=0$. Indeed

$$
\begin{aligned}
T_{P_{1}}(A) & =\varepsilon(A)\left(\sigma_{P_{1}}(A, B)+n+\sigma_{P_{1}}(A, B)\right) \\
& =\varepsilon(A) n \\
& =-\varepsilon(B) n \\
& =-T_{P_{1}}(B)
\end{aligned}
$$

where $n$ is an integer. Now we show $T_{P_{1}}(E)=T_{P_{2}}(E)$ for all $E \neq A, B$. If $\cdots E \cdots A B \cdots E \cdots B A \cdots$ or $\cdots A B \cdots E \cdots B A \cdots E$, then

$$
\begin{aligned}
T_{P_{1}}(E) & =\varepsilon(E)\left(n_{1}+\sigma_{P_{1}}(E, A)+\sigma_{P_{1}}(E, B)+n_{2}\right) \\
& =\varepsilon(E)\left(n_{1}+n_{2}\right) \\
& =T_{P_{2}}(E)
\end{aligned}
$$

where $n_{1}, n_{2}, n_{3}$ are integers If $\cdots E \cdots A B \cdots B A \cdots E \cdots$, then

$$
\begin{aligned}
T_{P_{1}}(E)=\varepsilon(E)\left(n_{1}+\sigma_{P_{1}}(E, A)+\right. & \sigma_{P_{1}}(E, B)+n_{2} \\
& \left.+\sigma_{P_{1}}(E, B)+\sigma_{P_{1}}(E, A)+n_{3}\right) \\
= & \varepsilon(E)\left(n_{1}+n_{2}+n_{3}\right) \\
= & T_{P_{2}}(E)
\end{aligned}
$$

where $n_{1}, n_{2}, n_{3}$ are integers. Therefore $T_{P_{1}}(E)=T_{P_{2}}(E)$ for all $E \neq A, B$.

Consider the 3-rd homotopy move $P_{1}:=\left(w_{1}|\cdots| x_{1} A B y_{1}|\cdots| x_{2} A C y_{2}|\cdots| x_{3} B C y_{3}|\cdots| w_{k}\right) \longrightarrow$

$$
P_{2}:=\left(w_{1}|\cdots| x_{1} B A y_{1}|\cdots| x_{2} C A y_{2}|\cdots| x_{3} C B y_{3}|\cdots| w_{k}\right)
$$

with $|A|=|B|=|C|$. In this case it is clear that $T\left(P_{1}\right)=T\left(P_{2}\right)$.

Consider the 3-rd homotopy move

$$
\begin{aligned}
& P_{1}:=\left(w_{1}|\cdots| x_{1} A B y_{1} A C z_{1}|\cdots| x_{2} B C y_{2}|\cdots| w_{k}\right) \longrightarrow \\
& \text { with }|A|=|B|=|C| .
\end{aligned}
$$


It is sufficient to show $T_{P_{1}}\left(x_{1} A B y_{1} A C z_{1}\right)=T_{P_{2}}\left(x_{1} B A y_{1} C A z_{1}\right)$ and $T_{P_{1}}\left(x_{2} B C y_{2}\right)$ $=T_{P_{2}}\left(x_{2} C B y_{2}\right)$.

$$
\begin{aligned}
T_{P_{1}}(A) & =\varepsilon(A)\left(\sigma_{P_{1}}(A, B)+n_{1}\right) \\
& =\varepsilon(A)\left(n_{1}+\sigma_{P_{2}}(A, C)\right) \\
& =T_{P_{2}}(A),
\end{aligned}
$$

where $n_{1}$ an integer. So $T_{P_{1}}\left(x_{1} A B y_{1} A C z_{1}\right)=T_{P_{2}}\left(x_{1} B A y_{1} C A z_{1}\right)$ holds.

$T_{P_{1}}\left(x_{2} B C y_{2}\right)=T_{P_{2}}\left(x_{2} C B y_{2}\right)$ is clear.

Consider the 3-rd homotopy move $P_{1}:=\left(w_{1}|\cdots| x_{1} A B y_{1}|\cdots| x_{2} A C y_{2} B C z_{2}|\cdots| w_{k}\right) \longrightarrow$

$$
P_{2}:=\left(w_{1}|\cdots| x_{1} B A y_{1}|\cdots| x_{2} C A y_{2} C B z_{2}|\cdots| w_{k}\right)
$$

with $|A|=|B|=|C|$. In this case $T\left(P_{1}\right)=T\left(P_{2}\right)$ is proved similarly to above case.

Consider the 3-rd homotopy move

$P_{1}:=\left(w_{1}|\cdots| x A B y A C z B C t|\cdots| w_{k}\right) \longrightarrow$

$$
P_{2}:=\left(w_{1}|\cdots| x B A y C A z C B t|\cdots| w_{k}\right)
$$

with $|A|=|B|=|C|$. In this case it is sufficient to show that $T_{P_{1}}(x A B y A C z B C t)$ $=T_{P_{2}}(x B A y C A z C B t) . T_{P_{1}}(A)=T_{P_{2}}(A)$ and $T_{P_{1}}(C)=T_{P_{2}}(C)$ is clear. Note that $\sigma_{P_{1}}(B, A)=-\sigma_{P_{1}}(B, C)$ and $\sigma_{P_{2}}(B, A)=\sigma_{P_{2}}(B, C)=0$. We obtain $T_{P_{1}}(B)=$ $T_{P_{2}}(B) . T_{P_{1}}(E)=T_{P_{2}}(E)$ for all $E \neq A, B, C$ is checked easily. So we obtain $T\left(P_{1}\right)=T\left(P_{2}\right)$.

Next we define invariant $T$ for nanophrases over one-point set. To define this invariant, we define some notation as followings.

Definition 3.9. Let $P:=\left(\mathcal{A},\left(w_{1}|\cdots| w_{k}\right)\right)$ be a nanophrase over one-point set $\alpha:=\{a\}$. Let $A, B \in \mathcal{A}$ be letters. Then we define $\tilde{\sigma}_{P}(A, B) \in \mathbb{Z} / 2 \mathbb{Z}$ as followings: If $A$ and $B$ forms $\cdots A \cdots B \cdots A \cdots B \cdots$ or $\cdots B \cdots A \cdots B \cdots A \cdots$ in $P$, then $\tilde{\sigma}_{P}(A, B):=1$. Otherwise $\tilde{\sigma}_{P}(A, B):=0$.

Definition 3.10. Let $P:=\left(\mathcal{A},\left(w_{1}|\cdots| w_{k}\right)\right)$ be a nanophrase over $\alpha:=\{a\}$. For $A \in \mathcal{A}$ such that there exist $i \in\{1,2, \cdots, k\}$ such that $\operatorname{Card}\left(w_{i}^{-1}(A)\right)=2$, we define $T_{P}(A) \in \mathbb{Z} / 2 \mathbb{Z}$ by

$$
T_{P}(A):=\sum_{B \in \mathcal{A}} \tilde{\sigma}_{P}(A, B) \in \mathbb{Z} / 2 \mathbb{Z},
$$

and $T_{P}\left(w_{i}\right) \in \mathbb{Z} / 2 \mathbb{Z}$ by

$$
T_{P}\left(w_{i}\right):=\sum_{A \in \mathcal{A}, \operatorname{Card}\left(w_{i}^{-1}(A)\right)=2} T_{P}(A) .
$$

Then we define $T(P) \in(\mathbb{Z} / 2 \mathbb{Z})^{k}$ by

$$
T(P):=\left(T_{P}\left(w_{1}\right), T_{P}\left(w_{2}\right), \cdots, T_{P}\left(w_{k}\right)\right) .
$$


Then next theorem follows.

Theorem 3.11. The $T$ is a homotopy invariant of nanophrases over one-point set.

Proof. Consider the 1-st homotopy move

$$
\begin{aligned}
P_{1}:=\left(w_{1}|\cdots| w_{l-1}|x A A y| w_{l+1} \mid\right. & \left.\cdots \mid w_{k}\right) \longrightarrow \\
& P_{2}:=\left(w_{1}|\cdots| w_{l-1}|x y| w_{l+1}|\cdots| w_{k}\right) .
\end{aligned}
$$

It is clear that $T_{P_{1}}\left(w_{i}\right)=T_{P_{2}}\left(w_{i}\right)$ for all $i \neq l$. We show that $T_{P_{1}}(x A A y)=T_{P_{2}}(x y)$. Note that $\tilde{\sigma}_{P_{1}}(A, B)=0$ for all $B \in \mathcal{A}$ by definition. Therefore $T_{P_{1}}(A)=0$. Moreover $\tilde{\sigma}_{P_{1}}(E, A)=0$ for all $E \in \mathcal{A}$. So $A$ does not contribute to $T_{P_{1}}(E)$ for all $E \in \mathcal{A}$. Therefore $T_{P_{1}}(x A A y)=T_{P_{2}}(x y)$.

Consider the 2-nd homotopy move such that $\mathrm{A}$ and $\mathrm{B}$ occur in some words once $P_{1}:=\left(w_{1}|\cdots| x_{1} A B y_{1}|\cdots| x_{2} B A y_{2}|\cdots| w_{k}\right) \longrightarrow$

$$
P_{2}:=\left(w_{1}|\cdots| x_{1} y_{1}|\cdots| x_{2} y_{2}|\cdots| w_{k}\right)
$$

with $|A|=\tau(|B|)$. It is sufficient to show that $T_{P_{1}}\left(x_{1} A B y_{1}\right)=T_{P_{2}}\left(x_{1} y_{1}\right)$ and $T_{P_{1}}\left(x_{2} B A y_{2}\right)=T_{P_{2}}\left(x_{2} y_{2}\right)$. Note that $A$ and $B$ occur in $P$ once. Moreover for all $E$ such that $\cdots E \cdots A B \cdots E \cdots$ in $P_{1}$

$$
\begin{aligned}
T_{P_{1}}(E) & =\varepsilon(E)\left(n_{1}+\tilde{\sigma}_{P_{1}}(E, A)+\tilde{\sigma}_{P_{1}}(E, B)+n_{2}\right) \\
& =\varepsilon(E)\left(n_{1}+2+n_{2}\right) \\
& =\varepsilon(E)\left(n_{1}+n_{2}\right) \\
& =T_{P_{2}}(E)
\end{aligned}
$$

where $n_{1}, n_{2}$ are integers. Therefore $T_{P_{1}}\left(x_{1} A B y_{1}\right)=T_{P_{2}}\left(x_{1} y_{1}\right) \cdot T_{P_{1}}\left(x_{2} B A y_{2}\right)=$ $T_{P_{2}}\left(x_{2} y_{2}\right)$ is proved similarly. The case of other type homotopy moves is proved similarly to above.

Remark 3.12. Any nanoword $w$ over $\alpha$ yields a nanophrase $(w)$ of length 1 . So we can consider the invariant of nanophrases over $\alpha_{0}$ (resp. one-point set) $T$ as a invariant of nanowords over $\alpha_{0}$ (resp. one-point set). But these invariants are useless. In fact it is easily checked that $T((w))=0$ for all nanowords over $\alpha_{0}$ and nanowords over one-point set.

\section{Classification of Nanophrases of Length 2 with 4 Letters.}

In this section we give the homotopy classification of nanophrases of length 2 less than 4 letters.

\subsection{Classification of nanophrases of length 2 with 2 letters.}

In this subsection we give the homotopy classification of nanowords of length 2 with 2 letter.

Consider a nanophrase of length 2 with 2 letter $P_{a}:=(A \mid A)$ with $|A|=a$. 
Theorem 4.1. Let $P$ be a nanophrase of length 2 with 2 letters. Then $P \nsucceq(\emptyset \mid \emptyset)$ if and only if $P \approx P_{a}$. Moreover $P_{a} \simeq P_{a^{\prime}}$ if and only if $a=a^{\prime}$.

Proof. The first part of this theorem is clear. We show the second part of this theorem. Suppose $P_{a} \simeq P_{a^{\prime}}$. Then $\gamma\left(P_{a}\right)=\gamma\left(P_{a^{\prime}}\right)$. This implies $z_{a} \otimes z_{\tau(a)}=$ $z_{a^{\prime}} \otimes z_{\tau\left(a^{\prime}\right)}$. Therefore $z_{a}=z_{a^{\prime}}$ in $\Pi$. It is possible only if $a=a^{\prime}$. So the theorem is proved.

\subsection{Classification of nanophrases of length 2 with 4 letters.}

First, we show following lemmas.

Lemma 4.2. Let $\beta$ be $\tau$-invariant subset of $\alpha$. If two nanophrases over $\beta$ are homotopic in the class of nanophrases over $\alpha$, then they are homotopic in the class of nanophrases over $\beta$.

Proof. This lemma is proved similarly to Lemma 2.6.

Lemma 4.3. Let $P_{1}=\left(w_{1}\left|w_{2}\right| \cdots \mid w_{k}\right)$ and $P_{2}=\left(v_{1}\left|v_{2}\right| \cdots \mid v_{k}\right)$ be nanophrases of length $k$ over $\alpha$. If $P_{1}$ and $P_{2}$ are homotopic as nanophrases, then $w_{1} w_{2} \cdots w_{k}$ and $v_{1} v_{2} \cdots v_{k}$ are homotopic as nanowords over $\alpha$.

Proof. It follows from definitions of homotopy of nanowords and homotopy of nanophrases.

Lemma 4.4. Let $P_{1}=\left(w_{1}\left|w_{2}\right| \cdots \mid w_{k}\right)$ and $P_{2}=\left(v_{1}\left|v_{2}\right| \cdots \mid v_{k}\right)$ be nanophrases of length $k$ over $\alpha$. If $P_{1}$ and $P_{2}$ are homotopic as nanophrases, then $w_{i}$ and $v_{i}$ are homotopic as étale words for all $i \in\{1,2, \cdots, k\}$.

Proof. This follows from the definition of homotopy moves and the desingularization of étale words.

The following lemma follows from the definition of homotopy moves of nanophrases.

Lemma 4.5. Let $P_{1}=\left(w_{1}|\cdots| w_{k}\right)$ and $P_{2}=\left(v_{1}|\cdots| v_{k}\right)$ are nanophrases of length $k$. If $P_{1}$ and $P_{2}$ are homotopic, then length of $w_{i}$ is equal to length of $v_{i}$ modulo 2 for all $i \in\{1,2, \cdots, k\}$.

Take two letters $a, b \in \alpha$ (possibly $a=b$ ). Let $\mathcal{A}$ be the $\alpha$-alphabet consisting the three letters $A, B$ with $|A|=a,|B|=b \in \alpha$. Consider the following nanophrases: $P_{a, b}^{4,0}:=(A B A B \mid \emptyset), P_{a, b}^{3,1}:=(A B A \mid B), P_{a, b}^{2,2 I}:=(A B \mid A B), P_{a, b}^{2,2 I I}:=(A B \mid B A)$, $P_{a, b}^{1,3}:=(A \mid B A B), P_{a, b}^{0,4}:=(\emptyset \mid A B A B)$. If $a=\tau(b)$, then $P_{a, b}^{4,0} \simeq P_{a, b}^{2,2 I} \simeq P_{a, b}^{2,2 I I} \simeq$ $P_{a, b}^{0,4} \simeq(\emptyset \mid \emptyset)$. So in this paper, if we write $P_{a, b}^{4,0}, P_{a, b}^{2,2 I}, P_{a, b}^{2,2 I I}, P_{a, b}^{0,4}$, then we always 
assume that $a \neq \tau(b)$. The following theorem gives the classification of nanophrases of length 2 with 4 letters.

Theorem 4.6. Let $P$ be a nanophrase of length 2 with 4 letters, then $P$ is either homotopic to nanophrases of length 2 with 2 letters or isomorphic to a nanophrase one of followings: $P_{a, b}^{4,0}, P_{a, b}^{3,1}, P_{a, b}^{2,2 I}, P_{a, b}^{2,2 I I}, P_{a, b}^{1,3}, P_{a, b}^{0,4}$. For $(i, j) \in$ $\{(4,0),(3,1),(2,2 I),(2,2 I I),(1,3),(0,4)\}$ and any $a, b \in \alpha$. The nanophrase $P_{a, b}^{i, j}$ is neither homotopic to $(\emptyset \mid \emptyset)$ nor homotopic to nanophrases of length 2 with 2 letters. The nanophrases $P_{a, b}^{i, j}$ and $P_{a^{\prime}, b^{\prime}}^{i, j}$ are homotopic if and only if $(a, b)=\left(a^{\prime}, b^{\prime}\right)$. For $(i, j) \neq\left(i^{\prime}, j^{\prime}\right)$, the nanophrases $P_{a, b}^{i, j}$ and $P_{a^{\prime}, b^{\prime}}^{i^{\prime}, j^{\prime}}$ are not homotopic for any $a, b, a^{\prime}, b^{\prime} \in \alpha$.

In [6], Turaev showed a stable equivalence class of an oriented, ordered, pointed multi-component curve on a surface is identified with a homotopy classes of a nanophrase in a 2-letter alphabet. So we obtain a following corollary.

Corollary 4.7. ([1]).

There are exactly 19 stable equivalence classes of two components pointed ordered, oriented, curves on surfaces with minimum crossing number $\leq 2$.

Proof of Theorem 4.6. The first claim of this theorem is clear. We prove latter part of this theorem.

Consider a nanophrase $P_{a, b}^{4,0} . P_{a, b}^{4,0} \not \subset(\emptyset \mid \emptyset)$ and $P_{a, b}^{4,0} \not P_{a^{\prime}}$ for any $a^{\prime} \in \alpha$ are follows from Lemma 4.5. $P_{a, b}^{4,0} \not P_{a^{\prime}, b^{\prime}}^{3,1}$ and $P_{a, b}^{4,0} \not P_{a^{\prime}, b^{\prime}}^{1,3}$ are follows from Lemma 4.5. $P_{a, b}^{4,0} \not P_{a^{\prime}, b^{\prime}}^{0,4}$ is follows from Lemma 4.4. Indeed the first étale word of $P_{a, b}^{4,0}$ is $A B A B$ and the first étale word of $P_{a^{\prime}, b^{\prime}}^{0,4}$ is $\emptyset$. $A B A B$ is not homotopic to $\emptyset$ by Theorem 2.7. ( Note that we assume $a \neq \tau(b)$ and $a^{\prime} \neq \tau\left(b^{\prime}\right)$ in this case ). $P_{a, b}^{4,0} \not P_{a^{\prime}, b^{\prime}}^{2,2 I I}$ follows from Lemma 4.3. Indeed a nanoword $A B B A$ with $|A|=a^{\prime},|B|=b^{\prime}$ is homotopic to $\emptyset$. On the other hand, a nanoword $A B A B$ with $|A|=a,|B|=b$ with $a \neq \tau(b)$ is not homotopic to $\emptyset$. Suppose that $P_{a, b}^{4,0} \simeq P_{a^{\prime}, b^{\prime}}^{2,2 I}$. Then $\gamma\left(P_{a, b}^{4,0}\right)=\gamma\left(P_{a^{\prime}, b^{\prime}}^{2,2 I}\right) \cdot \gamma\left(P_{a, b}^{4,0}\right)=$ $z_{a} z_{b} z_{\tau(a)} z_{\tau(b)} \otimes 1$ and $\gamma\left(P_{a^{\prime}, b^{\prime}}^{2,2 I}\right)=z_{a^{\prime}} z_{b^{\prime}} \otimes z_{\tau\left(a^{\prime}\right)} z_{\tau\left(b^{\prime}\right)}$. So $z_{\tau\left(a^{\prime}\right)} z_{\tau\left(b^{\prime}\right)}=1$. This implies $a^{\prime}=\tau\left(b^{\prime}\right)$. But this contradicts to $a^{\prime} \neq \tau\left(b^{\prime}\right)$. Therefore $P_{a, b}^{4,0} \nsucceq P_{a^{\prime}, b^{\prime}}^{2,2 I} . P_{a, b}^{4,0} \simeq P_{a^{\prime}, b^{\prime}}^{4,0}$ only if $(a, b)=\left(a^{\prime}, b^{\prime}\right)$ follows from Lemma 4.3 and Theorem 2.7.

Consider the nanophrase $P_{a, b}^{3,1} \cdot P_{a, b}^{3,1} \nsucceq(\emptyset \mid \emptyset)$ follows from Lemma 4.5. $P_{a, b}^{3,1} \nsucceq P_{a^{\prime}}$ is proved later. $P_{a, b}^{3,1} \not P_{a^{\prime}, b^{\prime}}^{2,2 I}$ and $P_{a, b}^{3,1} \not P_{a^{\prime}, b^{\prime}}^{2,2 I I}$ follows by Lemma 4.5. $P_{a, b}^{3,1} \not P_{a^{\prime}, b^{\prime}}^{1,3}$ is proved later. $P_{a, b}^{3,1} \not P_{a^{\prime}, b^{\prime}}^{0,4}$ follows from Lemma 4.5. Suppose $P_{3,1}^{a, b} \simeq P_{3,1}^{a^{\prime}, b^{\prime}}$. If $a \neq \tau(b)$, then $(a, b)=\left(a^{\prime}, b^{\prime}\right)$ by Theorem 2.7. If $a=\tau(b)$, then $a^{\prime}=\tau\left(b^{\prime}\right)$ by Theorem2.7 and Lemma 4.3. So $\gamma\left(P_{3,1}^{a, b}\right)=z_{a} z_{b} z_{\tau(a)} \otimes z_{\tau(b)}=z_{\tau(a)} \otimes z_{\tau(b)}$ and $\gamma\left(P_{3,1}^{a^{\prime}, b^{\prime}}\right)=z_{a}^{\prime} z_{b}^{\prime} z_{\tau\left(a^{\prime}\right)} \otimes z_{\tau\left(b^{\prime}\right)}=z_{\tau\left(a^{\prime}\right)} \otimes z_{\tau\left(b^{\prime}\right)}$. This implies $z_{\tau(a)}=z_{\tau\left(a^{\prime}\right)}$ and $z_{\tau(b)}=z_{\tau\left(b^{\prime}\right)}$. Therefore $(a, b)=\left(a^{\prime}, b^{\prime}\right)$.

Consider the nanophrase $P_{a, b}^{2,2 I} . P_{a, b}^{2,2 I} \nsucceq(\emptyset \mid \emptyset)$ and $P_{a, b}^{2,2 I} \nsucceq P_{a^{\prime}}$ follows from Lemma 4.3. $P_{a, b}^{2,2 I} \not P_{a^{\prime}, b^{\prime}}^{2,2 I I}$ follows from Lemma 4.3. $P_{a, b}^{2,2 I} \nsucceq P_{a^{\prime}, b^{\prime}}^{1,3}$ follows from 
Lemma4.5. Suppose $P_{a, b}^{2,2 I} \simeq P_{a^{\prime}, b^{\prime}}^{0,4}$. Then $\gamma\left(P_{a, b}^{2,2 I}\right)=\gamma\left(P_{a^{\prime}, b^{\prime}}^{0,4}\right)$. This implies $z_{a} z_{b}=$ 1. This is possible only if $a=\tau(b)$. But this contradicts to assumption. So $P_{a, b}^{2,2 I} \nsucceq$ $P_{a^{\prime}, b^{\prime}}^{0,4} \cdot P_{a, b}^{2,2 I} \simeq P_{a^{\prime}, b^{\prime}}^{2,2 I}$ if and only if $(a, b)=\left(a^{\prime}, b^{\prime}\right)$ follows by Lemma 4.3.

Consider the nanophrase $P_{a, b}^{2,2 I I}$. Suppose $P_{a, b}^{2,2 I I} \simeq(\emptyset \mid \emptyset)$. Then $\gamma\left(P_{a, b}^{2,2 I I}\right)=$ $\gamma((\emptyset \mid \emptyset))=1 \otimes 1$. This implies $z_{a} z_{b}=1$. So $a=\tau(b)$. But this contradicts to $a \neq \tau(b)$. Therefore $P_{a, b}^{2,2 I I} \neq(\emptyset \mid \emptyset) . P_{a, b}^{2,2 I I} \not P_{a^{\prime}}$ follows from Lemma 4.5. $P_{a, b}^{2,2 I I} \nsucceq$ $P_{a^{\prime}, b^{\prime}}^{1,3}$ follows from Lemma4.5. Suppose $P_{a, b}^{2,2 I I} \simeq P_{a^{\prime}, b^{\prime}}^{0,4}$. Then $\gamma\left(P_{a, b}^{2,2 I I}\right)=\gamma\left(P_{a^{\prime}, b^{\prime}}^{0,4}\right)$. This implies $z_{a} z_{b}=1$. This is possible only if $a=\tau(b)$. But this contradicts to assumption. So $P_{a, b}^{2,2 I I} \not P_{a^{\prime}, b^{\prime}}^{0,4}$. Suppose $P_{a, b}^{2,2 I I} \simeq P_{a^{\prime}, b^{\prime}}^{2,2 I I}$. Then $\gamma\left(P_{a, b}^{2,2 I I}\right)=$ $\gamma\left(P_{a^{\prime}, b^{\prime}}^{2,2 I I}\right)$. This implies to $z_{a} z_{b}=z_{a^{\prime}} z_{b^{\prime}}$. This is possible only if either " $a=a^{\prime}$ and $b=b^{\prime \prime}$ or " $a=\tau(b)$ and $a^{\prime}=\tau\left(b^{\prime}\right)$ ". The latter case contradicts to $a \neq \tau(b)$. So $(a, b)=\left(a^{\prime}, b^{\prime}\right)$. Therefore $P_{a, b}^{2,2 I I} \simeq P_{a^{\prime}, b^{\prime}}^{2,2 I}$ if and only if $(a, b)=\left(a^{\prime}, b^{\prime}\right)$.

Consider the nanophrase $P_{a, b}^{1,3} . P_{a, b}^{1,3} \not(\emptyset \mid \emptyset)$ follows from Lemma 4.5. $P_{a, b}^{1,3} \nsucceq P_{a^{\prime}}$ is proved later. $P_{a, b}^{1,3} \nsucceq P_{a^{\prime}, b^{\prime}}^{0,4}$ follows from Lemma 4.5. Suppose $P_{a, b}^{1,3} \simeq P_{a^{\prime}, b^{\prime}}^{1,3}$. If $a \neq \tau(b)$, then $(a, b)=\left(a^{\prime}, b^{\prime}\right)$ by Lemma 4.3 and Theorem 2.7. If $a=\tau(b)$, then $a^{\prime}=\tau\left(b^{\prime}\right)$. So if $\gamma\left(P_{a, b}^{1,3}\right)=\gamma\left(P_{a^{\prime}, b^{\prime}}^{1,3}\right)$, then $z_{a}=z_{a^{\prime}}$ and $z_{b}=z_{b^{\prime}}$. This implies $(a, b)=\left(a^{\prime}, b^{\prime}\right)$.

Consider the nanophrase $P_{a, b}^{0,4} . P_{a, b}^{0,4} \not(\emptyset \mid \emptyset)$ and $P_{a, b}^{0,4} \not P_{a^{\prime}}$ follow from Lemma 4.4. $P_{a, b}^{0,4} \simeq P_{a^{\prime}, b^{\prime}}^{0,4}$ if and only if $(a, b)=\left(a^{\prime}, b^{\prime}\right)$ follows from Lemma 4.3.

Now we proof following three remain parts of proof: $P_{a, b}^{3,1} \nsucc P_{a^{\prime}}, P_{a, b}^{3,1} \nsucc P_{a^{\prime}, b^{\prime}}^{1,3}$, and $P_{a, b}^{1,3} \nsucceq P_{a^{\prime}}$.

Suppose $P_{a, b}^{3,1} \simeq P_{a^{\prime}} \cdot \gamma\left(P_{a, b}^{3,1}\right)=z_{a} z_{b} z_{\tau(a)} \otimes z_{\tau(b)}$ and $\gamma\left(P_{a^{\prime}}\right)=z_{a^{\prime}} \otimes z_{\tau\left(a^{\prime}\right)}$. This implies $a^{\prime}=b$. Moreover $a=\tau(b)$ by Lemma 4.3. So if $a \neq b$, then $P_{a, b}^{3,1} \simeq P_{b}$ as nanophrases over $\alpha_{0}$. However,

$$
\begin{aligned}
T\left(P_{a, b}^{3,1}\right) & =\left(T_{P_{a, b}^{3,1}}(A B A), T_{P_{a, b}^{3,1}}(B)\right) \\
& =\left(\varepsilon(A) \sigma_{P_{a, b}^{3,1}}(A, B), 0\right) \\
& =(-1,0),
\end{aligned}
$$

and

$$
T\left(P_{b}\right)=(0,0) .
$$

This contradicts to homotopy invariance of $T$. If $a=b$, then $P_{a, a}^{3,1} \simeq P_{a}$ as nanophrases over $\alpha=\{a\}$. However

$$
\begin{gathered}
T\left(P_{a, a}^{3,1}\right)=(1,0) \in(\mathbb{Z} / 2 \mathbb{Z})^{2}, \\
T\left(P_{a}\right)=(0,0) \in(\mathbb{Z} / 2 \mathbb{Z})^{2} .
\end{gathered}
$$

This contradicts to homotopy invariance of $T$. Therefore $P_{a, b}^{3,1} \nsucceq P_{a^{\prime}}$.

$P_{a, b}^{1,3} \nsucceq P_{a^{\prime}}$ is proved similarly to above.

Suppose $P_{a, b}^{3,1} \simeq P_{a^{\prime}, b^{\prime}}^{1,3}$. If $a \neq \tau(b)$, then $a^{\prime} \neq \tau\left(b^{\prime}\right)$ and $(a, b)=\left(a^{\prime}, b^{\prime}\right)$ by Lemma 4.3. Moreover $\gamma\left(P_{a, b}^{1,3}\right)=\gamma\left(P_{a^{\prime}, b^{\prime}}^{1,3}\right)$ implies $z_{a} z_{b} z_{\tau(a)}=z_{a}$ and $z_{\tau(b)}=z_{b} z_{\tau(a)} z_{\tau(b)}$. So 
$z_{b} z_{\tau(a)}=1$ and this is possible only if $a=b$. Therefore $P_{a, a}^{3,1} \simeq P_{a, a}^{1,3}$ as nanowords over $\alpha_{0}=\{a, \tau(a)\}$ by Lemma 4.2. However,

$$
\begin{aligned}
T\left(P_{a, a}^{3,1}\right) & =\left(T_{P_{a, a}^{3, a}}(A B A), T_{P_{a, a}^{3,1}}(B)\right) \\
& =\left(\varepsilon(A) \sigma_{P_{a, a}^{3,1}}(A, B), 0\right) \\
& =(1,0),
\end{aligned}
$$

and

$$
\begin{aligned}
T\left(P_{a, a}^{1,3}\right) & =\left(T_{P_{a, a}^{1,3}}(A), T_{P_{a, a}^{1,3}}(B A B)\right) \\
& =\left(0, \varepsilon(B) \sigma_{P_{a, a}^{1,3}}(B, A)\right) \\
& =(0,-1) .
\end{aligned}
$$

This contradicts to homotopy invariance of $T$. If $a=\tau(b)$, then $a^{\prime}=\tau\left(b^{\prime}\right)$ by Lemma 4.3. Moreover $\gamma\left(P_{a, b}^{1,3}\right)=\gamma\left(P_{a^{\prime}, b^{\prime}}^{1,3}\right)$. This implies $z_{\tau(a)}=z_{a^{\prime}}$ and $z_{\tau(b)}=z_{b^{\prime}}$. So $a=\tau\left(a^{\prime}\right)$ and $b=\tau\left(b^{\prime}\right)$. If $a=\tau(a)$, then $a=a^{\prime}=b=b^{\prime}$ by above equations. Therefore $P_{a, a}^{3,1} \simeq P_{a, a}^{1,3}$ as nanophrases over $\alpha=\{a\}$. However,

$$
\begin{aligned}
& T\left(P_{a, a}^{3,1}\right)=(1,0) \in(\mathbb{Z} / 2 \mathbb{Z})^{2}, \\
& T\left(P_{a, a}^{1,3}\right)=(0,1) \in(\mathbb{Z} / 2 \mathbb{Z})^{2} .
\end{aligned}
$$

This contradicts to homotopy invariance of $T$. If $a \neq \tau(a)$, then $P_{a, b}^{3,1} \simeq P_{b, a}^{1,3}$ as nanophrases over $\alpha_{0}$. However,

$$
\begin{aligned}
& T\left(P_{a, b}^{3,1}\right)=\left(\varepsilon(A) \sigma_{P_{a, b}^{3,1}}(A, B), 0\right)=(-1,0) \\
& T\left(P_{b, a}^{1,3}\right)=\left(0, \varepsilon(B) \sigma_{P_{b, a}^{1,3}}(B, A)\right)=(0,1) .
\end{aligned}
$$

This contradicts to homotopy invariance of $T$. Therefore $P_{a, b}^{3,1} \nsucceq P_{a^{\prime}, b^{\prime}}^{1,3}$.

Now we have completed the homotopy classification of nanophrases of length 2 with 4 letters.

\section{References}

[1] T. Fukunaga, Topology of phrases and multi-components surface curves, Master Thesis, Hokkaido Univ., March 2008.

[2] C. F. Gauss, Werke, Vol.8, Teubner, Leipzig, 1900.

[3] N. Ito, Invariants via word for curves and fronts, math.GT/0705.0412.

[4] S. Kamada, Knot invariants derived from quandle and racks, Invariants of knots and 3-manifolds (Kyoto, 2001), 101-117 (electric).

[5] V. Turaev, Topology of words, math.CO/0503683.

[6] V. Turaev, Knots and words, International Mathematics Research Notices(2006), Art. ID 84098, 23 pp.

[7] V. Turaev, Lectures on topology of words, Japanese Journal of Mathematics.2(2007) 1-39.

[8] V. Turaev, Cobordisms of words, math.CO/0511513.

[9] V. Turaev, Virtual strings, Annals de l'Institut Fourier 54(2004), no.7, 2455-2525.

[10] V. Turaev, Curves on Surfaces, Charts, and Words , Geometriae Dedicata(2005), $116203-236$. 